\title{
NOTES ON THE SUPERSTABILITY OF D'ALEMBERT TYPE FUNCTIONAL EQUATIONS
}

\author{
Peng Cao and Bing Xu
}

\begin{abstract}
In this paper we will investigate the superstability of the generalized d'Alembert type functional equations $\sum_{i=1}^{m} f\left(x+\sigma^{i}(y)\right)=$ $k g(x) f(y)$ and $\sum_{i=1}^{m} f\left(x+\sigma^{i}(y)\right)=k f(x) g(y)$.
\end{abstract}

\section{Introduction}

D'Alembert functional equation

$$
f(x+y)+f(x-y)=2 f(x) f(y),
$$

also called the cosine functional equation, has a long history going back to J. D'Alembert [4]. The equation (1.1) plays an important role in determining the sum of two vectors in various Euclidean and non-Euclidean geometries.

The superstability of the d'Alembert functional equation (1.1) originated from J. A. Baker [2] under the condition $|f(x+y)+f(x-y)-2 f(x) f(y)| \leq \varepsilon$. R. Badora and R. Ger [1] generalized this result, by replacing $\varepsilon$ by $\varepsilon(x)$ or $\varepsilon(y)$. Recently, G. H. Kim investigated the superstability of the generalized d'Alembert type functional equations as follows:

$$
\begin{aligned}
& f(x+y)+f(x+\sigma y)=2 f(x) f(y), \\
& f(x+y)+f(x+\sigma y)=2 g(x) f(y), \\
& f(x+y)+f(x+\sigma y)=2 f(x) g(y) .
\end{aligned}
$$

Some special cases of the aforementioned functional equations were investigated (see, e.g., $[3,5,6,7,8,10,11])$.

The main purpose of this paper is to study the superstability of the more general d'Alembert type functional equations

$$
\sum_{i=1}^{m} f\left(x+\sigma^{i}(y)\right)=k g(x) f(y)
$$

Received April 3, 2008.

2000 Mathematics Subject Classification. 39B82, 39B32.

Key words and phrases. d'Alembert functional equation, superstability, cosine function.

(C)2009 The Korean Mathematical Society 


$$
\sum_{i=1}^{m} f\left(x+\sigma^{i}(y)\right)=k f(x) g(y)
$$

where $m$ is a positive integer and $k$ is an arbitrary nonzero complex number.

As an application, we obtain the superstability of functional equation

$$
\sum_{i=1}^{m} f\left(x+\sigma^{i}(y)\right)=k f(x) f(y) .
$$

In this paper, let $(\mathbf{G},+)$ be an Abelian group, denote by $\mathbb{N}, \mathbb{R}, \mathbb{C}$, as usual, the set of positive integer, the real and complex number field, respectively. Moreover, let $\sigma$ be an endomorphism of $\mathbf{G}$ with $\sigma^{m}(x)=x$. We may assume that $f$ and $g$ are nonzero functions, and $\varphi: \mathbf{G} \rightarrow \mathbb{R}$.

\section{Superstability of the equation (1.5)}

Theorem 1. Suppose that $f, g: \mathbf{G} \rightarrow \mathbb{C}$ satisfy the inequality

$$
\left|\sum_{i=1}^{m} f\left(x+\sigma^{i}(y)\right)-k g(x) f(y)\right| \leq\left\{\begin{array}{l}
\text { (i) } \varphi(x) \\
(\mathrm{ii}) \varphi(y) \text { and } \varphi(x)
\end{array}\right.
$$

for all $x, y \in \mathbf{G}$. Then

(i) either $f$ is bounded or $g$ satisfies (1.7),

(ii) either $g$ (or $f$ ) is bounded or $g$ satisfies (1.7),

also $f$ and $g$ satisfy (1.5) and (1.6).

Proof. For the case (i), let $f$ be unbounded. Then we can choose a sequence $\left(y_{n}\right)_{n>0}$ in $\mathbf{G}$ such that $\left|f\left(y_{n}\right)\right| \neq 0$ and

$$
\left|f\left(y_{n}\right)\right| \rightarrow \infty \quad \text { as } n \rightarrow \infty .
$$

We will show that $g$ satisfies (1.7). Taking $y=y_{n}$ in (i) of (2.1) we obtain

$$
\left|\frac{\sum_{i=1}^{m} f\left(x+\sigma^{i}\left(y_{n}\right)\right)}{k f\left(y_{n}\right)}-g(x)\right| \leq \frac{\varphi(x)}{\left|k f\left(y_{n}\right)\right|} \text {. }
$$

Taking the limit as $n \rightarrow \infty$, we obtain

$$
\lim _{n \rightarrow \infty} \frac{\sum_{i=1}^{m} f\left(x+\sigma^{i}\left(y_{n}\right)\right)}{k f\left(y_{n}\right)}=g(x)
$$

for all $x \in \mathbf{G}$. Using (i) of (2.1) we have

$$
\left|\sum_{j=1}^{m} \sum_{i=1}^{m} f\left(x+\sigma^{i}\left(y+\sigma^{j}\left(y_{n}\right)\right)\right)-k g(x) \sum_{j=1}^{m} f\left(y+\sigma^{j}\left(y_{n}\right)\right)\right| \leq m \varphi(x)
$$

for all $x, y \in \mathbf{G}$ and every $n \in \mathbb{N}$. Consider the condition $\sigma^{m}(x)=x$, we obtain

$$
\sum_{i=1}^{m} f\left(x+\sigma^{i}(y)\right) \equiv \sum_{i=1}^{m} f\left(x+\sigma^{s+i}(y)\right)
$$


for all $x, y \in \mathbf{G}$ and every $s \in \mathbb{N}$. Then by (2.5) and (2.4), we have

$$
\begin{aligned}
& \left|\sum_{i=1}^{m} \frac{\sum_{j=1}^{m} f\left(\left(x+\sigma^{i}(y)\right)+\sigma^{j}\left(y_{n}\right)\right)}{k f\left(y_{n}\right)}-k g(x) \frac{\sum_{j=1}^{m} f\left(y+\sigma^{j}\left(y_{n}\right)\right)}{k f\left(y_{n}\right)}\right| \\
= & \left|\sum_{i=1}^{m} \frac{\sum_{j=1}^{m} f\left(\left(x+\sigma^{i}(y)\right)+\sigma^{i+j}\left(y_{n}\right)\right)}{k f\left(y_{n}\right)}-k g(x) \frac{\sum_{j=1}^{m} f\left(y+\sigma^{j}\left(y_{n}\right)\right)}{k f\left(y_{n}\right)}\right| \\
= & \frac{\left|\sum_{j=1}^{m} \sum_{i=1}^{m} f\left(x+\sigma^{i}\left(y+\sigma^{j}\left(y_{n}\right)\right)\right)-k g(x) \sum_{j=1}^{m} f\left(y+\sigma^{j}\left(y_{n}\right)\right)\right|}{\left|k f\left(y_{n}\right)\right|} \\
\leq & \frac{m \varphi(x)}{\left|k f\left(y_{n}\right)\right|}
\end{aligned}
$$

for all $x, y \in \mathbf{G}$. By virtue of (2.3), we have

$$
\left|\sum_{i=1}^{m} g\left(x+\sigma^{i}(y)\right)-k g(x) g(y)\right|=0
$$

for all $x, y \in \mathbf{G}$. Therefore $g$ satisfies (1.7).

For the proof of the case (ii), first we show that $f$ (or $g$ ) is unbounded if and only if $g$ (or $f$ ) is also unbounded. Putting $y=0$ in (ii) of (2.1) we obtain

$$
|m f(x)-k g(x) f(0)| \leq \varphi(0)
$$

for all $x \in \mathbf{G}$. If $g$ is bounded, then by (2.6), we have

$$
|f(x)| \leq \frac{1}{m}|k g(x) f(0)|+\frac{1}{m} \varphi(0),
$$

which shows that $f$ is also bounded. On the other hand, if $f$ is bounded, we choose $y_{0} \in \mathbf{G}$ such that $f\left(y_{0}\right) \neq 0$, and then by (2.1) we obtain

$$
\left|\frac{\sum_{i=1}^{m} f\left(x+\sigma^{i}\left(y_{0}\right)\right)}{k f\left(y_{0}\right)}-g(x)\right| \leq \frac{\varphi\left(y_{0}\right)}{\left|k f\left(y_{0}\right)\right|}
$$

and it follows that $g$ is also bounded on $\mathbf{G}$.

Namely, if $f$ (or $g$ ) is unbounded, then so is $g$ (or $f$ ).

Let $g$ be unbounded, then $f$ is also unbounded. Then we can choose sequences $\left(x_{n}\right)_{n>0}$ and $\left(y_{n}\right)_{n>0}$ in $\mathbf{G}$ such that $\left|g\left(x_{n}\right)\right| \neq 0$ and $\left|g\left(x_{n}\right)\right| \rightarrow \infty$, $\left|f\left(y_{n}\right)\right| \neq 0$ and $\left|f\left(y_{n}\right)\right| \rightarrow \infty$ as $n \rightarrow \infty$.

Taking $x=x_{n}$ in (ii) of (2.1) we deduce

$$
\lim _{n \rightarrow \infty} \frac{\sum_{i=1}^{m} f\left(x_{n}+\sigma^{i}(y)\right)}{k g\left(x_{n}\right)}=f(y)
$$

for all $y \in \mathbf{G}$. Using (ii) of (2.1) we have

$$
\left|\sum_{i=1}^{m} \sum_{j=1}^{m} f\left(\left(x_{n}+\sigma^{i}(x)\right)+\sigma^{j}(y)\right)-k \sum_{i=1}^{m} g\left(x_{n}+\sigma^{i}(x)\right) f(y)\right| \leq m \varphi(y)
$$


for all $x, y \in \mathbf{G}$ and every $n \in \mathbb{N}$. Then by (2.5) and (2.8), we obtain

$$
\begin{aligned}
& \left|\sum_{j=1}^{m} \frac{\sum_{i=1}^{m} f\left(x_{n}+\sigma^{i}\left(x+\sigma^{j}(y)\right)\right)}{k g\left(x_{n}\right)}-k \frac{\sum_{i=1}^{m} g\left(x_{n}+\sigma^{i}(x)\right)}{k g\left(x_{n}\right)} f(y)\right| \\
= & \left|\frac{\sum_{i=1}^{m} \sum_{j=1}^{m} f\left(\left(x_{n}+\sigma^{i}(x)\right)+\sigma^{i+j}(y)\right)}{k g\left(x_{n}\right)}-k \frac{\sum_{i=1}^{m} g\left(x_{n}+\sigma^{i}(x)\right)}{k g\left(x_{n}\right)} f(y)\right| \\
= & \frac{\left|\sum_{i=1}^{m} \sum_{j=1}^{m} f\left(\left(x_{n}+\sigma^{i}(x)\right)+\sigma^{j}(y)\right)-k \sum_{i=1}^{m} g\left(x_{n}+\sigma^{i}(x)\right) f(y)\right|}{\left|k g\left(x_{n}\right)\right|} \\
\leq & \frac{m \varphi(y)}{\left|k g\left(x_{n}\right)\right|}
\end{aligned}
$$

for all $x, y \in \mathbf{G}$ and every $n \in \mathbb{N}$. Passing here to the limit as $n \rightarrow \infty$ with the use of $\left|g\left(x_{n}\right)\right| \rightarrow \infty$ and (2.7). Since $g$ satisfies (1.7) by (i), we have $\left|\sum_{j=1}^{m} f\left(x+\sigma^{j}(y)\right)-k g(x) f(y)\right|=0$, that is to say $f$ and $g$ are solutions of (1.5).

Applying (ii) of (2.1) again, we get

$$
\left|\sum_{i=1}^{m} \sum_{j=1}^{m} f\left(\left(x_{n}+\sigma^{i}(y)\right)+\sigma^{j}(x)\right)-k \sum_{i=1}^{m} g\left(x_{n}+\sigma^{i}(y)\right) f(x)\right| \leq m \varphi(x)
$$

and using (2.5), we have

$$
\begin{aligned}
& \left|\sum_{i=1}^{m} \frac{\sum_{j=1}^{m} f\left(x_{n}+\sigma^{j}\left(x+\sigma^{i}(y)\right)\right)}{k g\left(x_{n}\right)}-k f(x) \frac{\sum_{i=1}^{m} g\left(x_{n}+\sigma^{i}(y)\right)}{k g\left(x_{n}\right)}\right| \\
= & \left|\sum_{j=1}^{m} \frac{\sum_{i=1}^{m} f\left(\left(x_{n}+\sigma^{j}(x)\right)+\sigma^{j+i}(y)\right)}{k g\left(x_{n}\right)}-k f(x) \frac{\sum_{i=1}^{m} g\left(x_{n}+\sigma^{i}(y)\right)}{k g\left(x_{n}\right)}\right| \\
= & \left|\sum_{j=1}^{m} \frac{\sum_{i=1}^{m} f\left(\left(x_{n}+\sigma^{j}(x)\right)+\sigma^{i}(y)\right)}{k g\left(x_{n}\right)}-k f(x) \frac{\sum_{i=1}^{m} g\left(x_{n}+\sigma^{i}(y)\right)}{k g\left(x_{n}\right)}\right| \\
= & \frac{\left|\sum_{i=1}^{m} \sum_{j=1}^{m} f\left(\left(x_{n}+\sigma^{i}(y)\right)+\sigma^{j}(x)\right)-k f(x) \sum_{i=1}^{m} g\left(x_{n}+\sigma^{i}(y)\right)\right|}{\left|k g\left(x_{n}\right)\right|} \\
\leq & \frac{m \varphi(x)}{\left|k g\left(x_{n}\right)\right|}
\end{aligned}
$$

for all $x, y \in \mathbf{G}$ and every $n \in \mathbb{N}$. Using (2.7) and the fact that $g$ satisfies (1.7) by (i), we have $\left|\sum_{i=1}^{m} f\left(x+\sigma^{i}(y)\right)-k f(x) g(y)\right|=0$, that is to say $f$ and $g$ are solutions of (1.6). 
In the case of $m=k=2$ in Theorem 1, we can obtain the following corollary, which was investigated by G. H. Kim [9].

Corollary 1 ([9]). Suppose that $f, g: \mathbf{G} \rightarrow \mathbb{C}$ satisfy the inequality

$$
|f(x+y)+f(x+\sigma(y))-2 g(x) f(y)| \leq\left\{\begin{array}{l}
(\mathrm{i}) \varphi(x) \\
(\mathrm{ii}) \varphi(y) \text { and } \varphi(x)
\end{array}\right.
$$

for all $x, y \in \mathbf{G}$. Then

(i) either $f$ is bounded or $g$ satisfies (1.2),

(ii) either $g$ (or $f)$ is bounded or $g$ satisfies (1.2),

also $f$ and $g$ satisfy (1.3) and (1.4).

If $g=f$ in Theorem 1 , then the stability problem of the functional equation (1.7) is proved as a corollary.

Corollary 2. Suppose that $f: \mathbf{G} \rightarrow \mathbb{C}$ satisfies the inequality

$$
\left|\sum_{i=1}^{m} f\left(x+\sigma^{i}(y)\right)-k f(x) f(y)\right| \leq\left\{\begin{array}{l}
\text { (i) } \varphi(x) \\
\text { (ii) } \varphi(y) \text { and } \varphi(x)
\end{array}\right.
$$

for all $x, y \in \mathbf{G}$. Then, in all cases (i) and (ii), either $f$ is bounded or $f$ satisfies $(1.7)$.

\section{Superstability of the equation (1.6)}

We will prove the stability of (1.6) using a strategy similar to that of Theorem 1.

Theorem 2. Suppose that $f, g: \mathbf{G} \rightarrow \mathbb{C}$ satisfy the inequality

$$
\left|\sum_{i=1}^{m} f\left(x+\sigma^{i}(y)\right)-k f(x) g(y)\right| \leq\left\{\begin{array}{l}
(\mathrm{i}) \varphi(y) \\
(\mathrm{ii}) \varphi(x) \text { and } \varphi(y)
\end{array}\right.
$$

for all $x, y \in \mathbf{G}$. Then

(i) either $f$ is bounded or $g$ satisfies (1.7),

(ii) either $g$ (or $f)$ with $f(\sigma x)=f(x)$ is bounded or $g$ satisfies (1.7), also $f$ and $g$ satisfy (1.5) and (1.6).

Proof. For the case (i), let $f$ be unbounded. Then we can choose a sequence $\left(x_{n}\right)_{n>0}$ in $\mathbf{G}$ such that $\left|f\left(x_{n}\right)\right| \neq 0$ and

$$
\left|f\left(x_{n}\right)\right| \rightarrow \infty \quad \text { as } \quad n \rightarrow \infty .
$$

We will show that $g$ satisfies (1.7). Taking $x=x_{n}$ in (3.1) we obtain

$$
\lim _{n \rightarrow \infty} \frac{\sum_{i=1}^{m} f\left(x_{n}+\sigma^{i}(y)\right)}{k f\left(x_{n}\right)}=g(y)
$$


for all $y \in \mathbf{G}$. Using (i) of (3.1) we have

$$
\left|\sum_{i=1}^{m} \sum_{j=1}^{m} f\left(\left(x_{n}+\sigma^{i}(x)\right)+\sigma^{j}(y)\right)-k \sum_{i=1}^{m} f\left(x_{n}+\sigma^{i}(x)\right) g(y)\right| \leq m \varphi(y)
$$

for all $x, y \in \mathbf{G}$ and every $n \in \mathbb{N}$. Then by (2.5) and (3.4), we obtain

$$
\begin{aligned}
& \left|\sum_{j=1}^{m} \frac{\sum_{i=1}^{m} f\left(x_{n}+\sigma^{i}\left(x+\sigma^{j}(y)\right)\right)}{k f\left(x_{n}\right)}-k \frac{\sum_{i=1}^{m} f\left(x_{n}+\sigma^{i}(x)\right)}{k f\left(x_{n}\right)} g(y)\right| \\
= & \left|\sum_{i=1}^{m} \frac{\sum_{j=1}^{m} f\left(\left(x_{n}+\sigma^{i}(x)\right)+\sigma^{i+j}(y)\right)}{k f\left(x_{n}\right)}-k \frac{\sum_{i=1}^{m} f\left(x_{n}+\sigma^{i}(x)\right)}{k f\left(x_{n}\right)} g(y)\right| \\
= & \frac{\left|\sum_{i=1}^{m} \sum_{j=1}^{m} f\left(\left(x_{n}+\sigma^{i}(x)\right)+\sigma^{j}(y)\right)-k \sum_{i=1}^{m} f\left(x_{n}+\sigma^{i}(x)\right) g(y)\right|}{\left|k f\left(x_{n}\right)\right|} \\
\leq & \frac{m \varphi(y)}{\left|k f\left(x_{n}\right)\right|}
\end{aligned}
$$

for all $x, y \in \mathbf{G}$. By virtue of (3.2) and (3.3), we have

$$
\left|\sum_{j=1}^{m} g\left(x+\sigma^{j}(y)\right)-k g(x) g(y)\right|=0
$$

for all $x, y \in \mathbf{G}$. Therefore $g$ satisfies (1.7).

For the case (ii), we can see that, similar to Theorem 1, $f$ (or $g$ ) is unbounded if and only if $g$ (or $f$ ) is also unbounded. Namely, if $f$ is bounded, choose $x_{0} \in \mathbf{G}$ such that $f\left(x_{0}\right) \neq 0$ and use (ii) of (3.1) to get

$$
|g(y)|-\frac{\left|\sum_{i=1}^{m} f\left(x_{0}+\sigma^{i}(y)\right)\right|}{\left|k f\left(x_{0}\right)\right|} \leq\left|\frac{\sum_{i=1}^{m} f\left(x_{0}+\sigma^{i}(y)\right)}{k f\left(x_{0}\right)}-g(y)\right| \leq \frac{\varphi\left(x_{0}\right)}{\left|k f\left(x_{0}\right)\right|},
$$

which shows that $g$ is also bounded.

Suppose $f$ is unbounded. Putting $x=0$ in (ii) of (3.1), we have

$$
\left|\sum_{i=1}^{m} f\left(\sigma^{i} y\right)-k f(0) g(y)\right| \leq \varphi(0)
$$

that is, $|m f(y)-k f(0) g(y)| \leq \varphi(0)$, since $f(\sigma x)=f(x)$ for all $x \in \mathbf{G}$. This implies that $g$ is also unbounded.

Let $g$ be unbounded, then $f$ is also unbounded. Then we can choose sequences $\left(x_{n}\right)_{n>0}$ and $\left(y_{n}\right)_{n>0}$ in $\mathbf{G}$ such that $\left|f\left(x_{n}\right)\right| \neq 0$ and $\left|f\left(x_{n}\right)\right| \rightarrow \infty$, $\left|g\left(y_{n}\right)\right| \neq 0$ and $\left|g\left(y_{n}\right)\right| \rightarrow \infty$ as $n \rightarrow \infty$.

Taking $y=y_{n}$ in (ii) of (3.1) we deduce

$$
\lim _{n \rightarrow \infty} \frac{\sum_{i=1}^{m} f\left(x+\sigma^{i}\left(y_{n}\right)\right)}{k g\left(y_{n}\right)}=f(x)
$$


for all $x \in \mathbf{G}$. Using (ii) of (3.1) we have

$$
\left|\sum_{j=1}^{m} \sum_{i=1}^{m} f\left(x+\sigma^{i}\left(y+\sigma^{j}\left(y_{n}\right)\right)\right)-k f(x) \sum_{j=1}^{m} g\left(y+\sigma^{j}\left(y_{n}\right)\right)\right| \leq m \varphi(x)
$$

for all $x, y \in \mathbf{G}$ and every $n \in \mathbb{N}$. Then by (2.5) and (3.6), we obtain

$$
\begin{aligned}
& \left|\sum_{i=1}^{m} \frac{\sum_{j=1}^{m} f\left(\left(x+\sigma^{i}(y)\right)+\sigma^{j}\left(y_{n}\right)\right)}{k g\left(y_{n}\right)}-k f(x) \frac{\sum_{j=1}^{m} g\left(y+\sigma^{j}\left(y_{n}\right)\right)}{k g\left(y_{n}\right)}\right| \\
= & \left|\sum_{i=1}^{m} \frac{\sum_{j=1}^{m} f\left(\left(x+\sigma^{i}(y)\right)+\sigma^{i+j}\left(y_{n}\right)\right)}{k g\left(y_{n}\right)}-k f(x) \frac{\sum_{j=1}^{m} g\left(y+\sigma^{j}\left(y_{n}\right)\right)}{k g\left(y_{n}\right)}\right| \\
= & \frac{\left|\sum_{j=1}^{m} \sum_{i=1}^{m} f\left(x+\sigma^{i}\left(y+\sigma^{j}\left(y_{n}\right)\right)\right)-k f(x) \sum_{j=1}^{m} g\left(y+\sigma^{j}\left(y_{n}\right)\right)\right|}{\left|k g\left(y_{n}\right)\right|} \\
\leq & \frac{m \varphi(x)}{\left|k g\left(y_{n}\right)\right|}
\end{aligned}
$$

for all $x, y \in \mathbf{G}$. Since $g$ satisfies (1.7), it follows from (3.5) that

$$
\left|\sum_{i=1}^{m} f\left(x+\sigma^{i}(y)\right)-k f(x) g(y)\right|=0
$$

for all $x, y \in \mathbf{G}$. Hence $f$ and $g$ are solutions of (1.6).

Applying (ii) of (3.1) again, we get

$$
\left|\sum_{j=1}^{m} \sum_{i=1}^{m} f\left(y+\sigma^{i}\left(x+\sigma^{j}\left(y_{n}\right)\right)\right)-k f(y) \sum_{j=1}^{m} g\left(x+\sigma^{j}\left(y_{n}\right)\right)\right| \leq m \varphi(y)
$$

for all $x, y \in \mathbf{G}$. Since $f(\sigma x)=f(x)$ for all $x \in \mathbf{G}$ and (3.7), we have

$$
\begin{aligned}
& \left|\sum_{i=1}^{m} \frac{\sum_{j=1}^{m} f\left(\left(x+\sigma^{m-i}(y)\right)+\sigma^{j}\left(y_{n}\right)\right)}{k g\left(y_{n}\right)}-k \frac{\sum_{j=1}^{m} g\left(x+\sigma^{j}\left(y_{n}\right)\right)}{k g\left(y_{n}\right)} f(y)\right| \\
= & \left|\frac{\sum_{i=1}^{m} \sum_{j=1}^{m} f\left(\sigma^{i}\left(x+\sigma^{m-i}(y)+\sigma^{j}\left(y_{n}\right)\right)\right)}{k g\left(y_{n}\right)}-k \frac{\sum_{j=1}^{m} g\left(x+\sigma^{j}\left(y_{n}\right)\right)}{k g\left(y_{n}\right)} f(y)\right| \\
= & \frac{\left|\sum_{j=1}^{m} \sum_{i=1}^{m} f\left(y+\sigma^{i}\left(x+\sigma^{j}\left(y_{n}\right)\right)\right)-k \sum_{j=1}^{m} g\left(x+\sigma^{j}\left(y_{n}\right)\right) f(y)\right|}{\left|k g\left(y_{n}\right)\right|} \\
\leq & \frac{m \varphi(y)}{\left|k g\left(y_{n}\right)\right|}
\end{aligned}
$$


for all $x, y \in \mathbf{G}$. Since $g$ satisfies (1.7), using (3.5), we have

$$
\left|\sum_{i=1}^{m} f\left(x+\sigma^{i}(y)\right)-k g(x) f(y)\right|=\left|\sum_{i=1}^{m} f\left(x+\sigma^{m-i}(y)\right)-k g(x) f(y)\right|=0
$$

for all $x, y \in \mathbf{G}$. Therefore $f$ and $g$ are solutions of (1.5).

In the case of $m=k=2$ in Theorem 2, we can obtain the following corollary, which was investigated by G. H. Kim [9].

Corollary $3([9])$. Suppose that $f, g: \mathbf{G} \rightarrow \mathbb{C}$ satisfy the inequality

$$
|f(x+y)+f(x+\sigma(y))-2 f(x) g(y)| \leq\left\{\begin{array}{l}
(\mathrm{i}) \varphi(y) \\
(\mathrm{ii}) \varphi(x) \text { and } \varphi(y)
\end{array}\right.
$$

for all $x, y \in \mathbf{G}$. Then

(i) either $f$ is bounded or $g$ satisfies (1.2),

(ii) either $g$ (or $f)$ with $f(\sigma x)=f(x)$ is bounded or $g$ satisfies (1.2), also $f$ and $g$ satisfy (1.3) and (1.4).

If we apply the case $g=f$ to Theorem 2 , then the stability problem of the functional equation (1.7) is proved as a corollary.

Corollary 4. Suppose that $f: \mathbf{G} \rightarrow \mathbb{C}$ satisfies the inequality

$$
\left|\sum_{i=1}^{m} f\left(x+\sigma^{i}(y)\right)-k f(x) f(y)\right| \leq\left\{\begin{array}{l}
(\mathrm{i}) \varphi(y) \\
\text { (ii) } \varphi(x) \text { and } \varphi(y)
\end{array}\right.
$$

for all $x, y \in \mathbf{G}$. Then, in all cases (i) and (ii), either $f$ is bounded or $f$ satisfies (1.7).

Example. Let $\sigma(x)=a x$, where $a$ is a constant in $\mathbb{C}$ satisfying $a^{m}=1$. Then Theorem 1 and Theorem 2 hold for the functional equations

$$
\begin{aligned}
& \sum_{i=1}^{m} f\left(x+a^{i} y\right)=k g(x) f(y), \\
& \sum_{i=1}^{m} f\left(x+a^{i} y\right)=k f(x) g(y) .
\end{aligned}
$$

\section{References}

[1] R. Badora and R. Ger, On some trigonometric functional inequalities, Functional equations-results and advances, 3-15, Adv. Math. (Dordr.), 3, Kluwer Acad. Publ., Dordrecht, 2002.

[2] J. A. Baker, The stability of the cosine equation, Proc. Amer. Math. Soc. 80 (1980), no. $3,411-416$.

[3] J. Baker, J. Lawrence, and F. Zorzitto, The stability of the equation $f(x+y)=f(x) f(y)$, Proc. Amer. Math. Soc. 74 (1979), no. 2, 242-246.

[4] J. D'Alembert, Mémoire sur les principes de la mécanique, Hist. Acad. Sci. (1769), $278-286$. 
[5] D. H. Hyers, G. Isac, and Th. M. Rassias, Stability of Functional Equations in Several Variables, Progress in Nonlinear Differential Equations and their Applications, 34. Birkhauser Boston, Inc., Boston, MA, 1998.

[6] S.-M. Jung, On an asymptotic behavior of exponential functional equation, Acta Math. Sin. (Engl. Ser.) 22 (2006), no. 2, 583-586.

[7] Pl. Kannappan, The functional equation $f(x y)+f\left(x y^{-1}\right)=2 f(x) f(y)$ for groups, Proc. Amer. Math. Soc. 19 (1968), no. 1, 69-74.

[8] Pl. Kannappan and G. H. Kim, On the stability of the generalized cosine functional equations, Ann. Acad. Paedagogicae Cracoviensis-Studia Mathematica 1 (2001), 49-58.

[9] G. H. Kim, The stability of d'Alembert and Jensen type functional equations, J. Math. Anal. Appl. 325 (2007), no. 1, 237-248.

[10] A. Redouani, E. Elqorachi, and Th. M. Rassias, The superstability of d'Alembert's functional equation on step 2 nilpotent groups, Aequationes Math. 74 (2007), no. 3, 226-241.

[11] Th. M. Rassias, On the stability of functional equations and a problem of Ulam, Acta Appl. Math. 62 (2000), no. 1, 23-130.

PENG CAO

Department of Mathematics

Sichuan University

Chengdu, Sichuan 610064, China

E-mail address: carol8253@126.com

Bing Xu

Department of Mathematics

Sichuan University

Chengdu, Sichuan 610064, China 